\title{
Drivers vs. Inhibitors - What Clinches Continuous Service Certification Adoption by Cloud Service Providers?
}

\author{
Heiner Teigeler, Sebastian Lins, Ali Sunyaev \\ Information Systems and Systems Engineering \\ Research Center for IS Design (ITeG) \\ University of Kassel, Germany \\ \{teigeler, lins, sunyaev\}@,uni-kassel.de
}

\begin{abstract}
Continuous service certification (CSC) is an innovative way to ensure ongoing security and reliability of cloud services by using (automated) monitoring and auditing techniques. Yet, CSC currently remains underexplored and is still in its early diffusion period, thus we require a deeper understanding about what influences cloud service providers to participate in CSC. In particular, cloud service providers are facing a trade-off between achieving unique advantages and participating in a complex and effortful CSC process. This study derives a theoretical model grounded on the Technology-Organization-Environment Framework and Diffusion of Innovations Theory to explain why cloud service providers might be willing to adopt CSC. To test our model, we surveyed 115 cloud service providers. Our findings reveal that perceived advantages have a significant influence on provider's adoption intention and that they outweigh perceived inhibitors.
\end{abstract}

\section{Introduction}

Cloud service providers often host critical data and processes for organizations that consume offered cloud services [24]. When outsourcing these parts to cloud service providers, organizations have a lack of control over their own data as their data is physically stored in the datacenters of their providers. To counteract this problem there are already several certification approaches that assure secure and reliable cloud services by independent third parties [28]. To support their daily IT needs for critical processes, the necessity for continuous, highly reliable and secure services from a customer's perspective gains high importance. Cloud service providers can participate in continuous service certification (CSC) to show that they continuously comply with defined cloud standards and requirements regarding, for example, data security, privacy and availability $[12,13,15]$.
CSC builds on (automated) monitoring and auditing by an independent third party to continuously check service provider's adherence to certification requirements [13]. Yet, CSC currently remains underexplored and evaluated in trials because innovative CSC process and architecture models, and corresponding prototypes were invented and developed during research projects only, thus CSC is in its early diffusion period [14].

When deciding to adopt CSC, providers face a tradeoff between achieving unique advantages and participating in a complex and effortful CSC process [14]. For example, when participating in CSC, providers receive ongoing and valuable feedback about their cloud services by an independent third party. Providers can reduce costs through successive service improvements, improved customer support, and fewer individual customer audits. Finally, by offering customers more transparent services, providers differentiate themselves in the market and gain a competitive advantage. These advantages will increase provider's intention to adopt CSC.

Besides its many advantages, providers will also face adoption inhibitors due to a complex and effortful CSC process. CSC adoption requires providers to set up a comprehensive monitoring and auditing infrastructure. Providers need to implement large-scale (continuous) monitoring systems to ensure that all audit-relevant data is available, up-to-date and accurate, and more importantly, can be transmitted to cloud service auditors to analyze certification requirement adherence. This leads to a high degree of complexity and requires a high technical knowledge for meeting hard- and software requirements. In addition, a low compatibility to the existing cloud infrastructure might act as an additional inhibitor in case of CSC adoption. These issues will ultimately hamper the adoption of CSC. Nevertheless, cloud service providers are still uncertain, whether the unique advantages might still outweigh the perceived expenses and efforts of participating in a complex CSC. Thus, we lack an understanding about how the aforementioned advantages and inhibitors will actually influence cloud providers' adoption intention. 
Reviewing literature on CSC in the context of cloud services as well as prior research on related continuous assessment approaches reveals that most studies focus on how to achieve and implement continuous assessment approaches by developing and evaluating technological and organizational means (e.g., in the context of ERP systems [25], web services [34], and cloud computing [13]). In contrast, prior literature has mostly neglected to understand why stakeholders (i.e., certification authorities, auditors and service providers) are actually willing to adopt those continuous assessment approaches. For example, researchers have analyzed why auditors are willing to use computer-assisted audit tools and techniques to increase their assessment efficiency $[1,2]$, as well as surveyed internal auditors' usage of continuous auditing technologies [32]. While previous adoption research has mostly focused on understanding internal auditors' adoption intention of continuous assessments approaches, we lack a deep understanding why cloud service providers are clinched to adopt continuous assessment approaches like CSC. As CSC exhibits unique characteristics, including an independent third party that performs the continuous assessments, a high technological complexity and a high observability of CSC results, applying previous research findings in CSC contexts might be limited. Consequently, it remains unclear why providers are driven to adopt CSC. To address this problem, we will answer the following research question with our study:

RQ: Which drivers or inhibitors influence cloud service providers to adopt CSC?

To evaluate factors that might influence the adoption of CSC in the context of cloud providers, this study develops and tests a theoretical model based on the Technology-Organization-Environment (TOE) framework and the Diffusion of Innovations (DOI) theory by conducting a survey among 115 cloud service providers.

Our findings confirm that cloud service providers are willing to adopt CSC. In particular, our results show that perceived advantages strongly urge cloud service providers to adopt CSC. In regard to the impact of perceived inhibitors, our survey revealed a surprising finding as none of the factors are significant, and thus advantages outweigh potential inhibitors.

We contribute to research by deriving and testing a theoretical model explaining the CSC adoption intention of service providers, ultimately deepening the knowledge about what drives providers' adoption intention. For practice, our study highlights that certification authorities should promote unique advantages of their CSC services to motivate cloud service providers to participate in CSC.

Next, we provide information about CSC, related work and relevant adoption theories. In Section 3, we define our research model. In Section 4, we outline our research approach and present our data analysis results. The next section discusses our findings and implications, followed by a conclusion of our work.

\section{Theoretical Background}

\subsection{Continuous Cloud Service Certification}

Existing cloud service certifications represent only a retrospective look at the fulfilment of technical and organizational measures at the time of their issuing [12]. Conditions and requirements of certifications may no longer be met throughout these validity periods because cloud services are confronted with an ever-changing environment. As such, inherent cloud computing characteristics (e.g., entangled supply chains), fast technology life cycles leading to ongoing architectural changes, the emergence of environmental threats (i.e., new software vulnerabilities) or changes in legal and regulatory landscape might have an impact on long-term certification reliability [13]. Just recently, information systems and computer science researchers have started to investigate how to innovate the process of certifying cloud services to cope with these challenges of an ever-changing and dynamic cloud service environment and to increase certification reliability (e.g., $[13,27])$. These research efforts resulted in innovative specifications of architectures and processes as well as prototypes that allow certification authorities to continuously certify cloud service providers. A CSC process comprises automated monitoring and auditing techniques as well as mechanisms for a transparent provision of certification-relevant information [12]. Yet, CSC currently remains underexplored and evaluated in trials only as CSC process and architecture models, and corresponding prototypes were mostly invented and developed during research projects [14]. Thus, CSC is still on its early diffusion period. Studying how to motivate relevant stakeholders to adopt CSC gains high importance to achieve aforementioned advantages of CSC, increase the maturity of CSC processes and (prototype) systems, and to pave the way for continuously reliable and secure cloud services. In this study, we focus on what drives cloud service providers to adopt CSC because providers are needed to create a market demand of CSC. When cloud service providers are willing to adopt CSC, certification authorities might start to set up new business models and serve the cloud market by providing innovative CSC services.

\subsection{Related Work}

Reviewing literature on CSC in the context of cloud services as well as prior research on related continuous assessment approaches reveals that most studies focus 
Table 1. Related literature on continuous assessments of information systems

\begin{tabular}{|c|c|c|c|}
\hline & & \multicolumn{2}{|c|}{ Continuous assessments context } \\
\hline & & Information Systems & Cloud Services \\
\hline \multirow{2}{*}{ 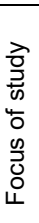 } & $\begin{array}{l}\text { Evaluate } \\
\text { means for con- } \\
\text { tinuous assess- } \\
\text { ments }\end{array}$ & $\begin{array}{l}\text { A Typical research question: „How can a contin- } \\
\text { uous assessment systems be designed?” } \\
\text { Exemplary Studies: }[25,34]\end{array}$ & $\begin{array}{l}\text { B Typical research question: „How can continuous } \\
\text { certification of cloud services be achieved?” } \\
\text { Exemplary Studies: }[13-15,27]\end{array}$ \\
\hline & $\begin{array}{l}\text { Understanding } \\
\text { stakeholders' } \\
\text { adoption }\end{array}$ & $\begin{array}{l}\text { C Typical research question: „Are organizations } \\
\text { adopting continuous assessment systems?” } \\
\text { Exemplary Studies: }[1,2]\end{array}$ & $\begin{array}{l}\text { D Typical research question: "What influences } \\
\text { stakeholders to adopt CSC?” } \\
\text { Exemplary Studies: This study }\end{array}$ \\
\hline
\end{tabular}

on how to achieve and implement continuous assessment approaches by developing and evaluating technological and organizational means. In contrast, prior literature has mostly neglected to understand why stakeholders are actually willing to adopt those assessment approaches. Table 1 separates related work based on their study focus (developing technological and organizational means to enable continuous assessments; or understanding stakeholder adoption) and the continuous assessments context (general information systems context; or cloud service context). Based on this separation quadrant A summarizes most of the related work that focuses on developing continuous assessment means. These studies have developed and evaluated technical concepts (i.e., embedded audit modules and digital agents) about (continuous) third party assessments in general, for example, in the context of certification of web services [34] and ERP systems [25]. Quadrant B shows that researchers have already started to develop and evaluate technical and organizational means to continuously assess cloud services as well. In particular, related studies are focusing on developing concepts, frameworks and prototypes to enable certification authorities to assess cloud services on a continuous basis $[12-15,27]$. In regard to understanding why stakeholders are willing to adopt such assessment approaches, several studies have recently started to analyze auditors' adoption intention in an information systems context (see quadrant C) $[1,2]$. For example, researchers have analyzed why auditors are willing to use computer-assisted audit tools and techniques to increase their assessment efficiency [2], as well as surveyed internal auditors' usage of continuous auditing technologies [32]. Yet previous adoption research has mostly focused on understanding internal auditors' adoption intention of continuous assessments approaches. To the best of our knowledge, prior literature has neglected to understand why service providers are willing to adopt CSC, in the context of cloud services in particular. As CSC exhibits unique characteristics, including an independent third party that performs the continuous assessments, a high technological complexity and a high observability of CSC results, applying previous research findings in CSC contexts might be limited, and requires further investigation. To address this gap in literature, this study focuses on the adoption of CSC and is located in quadrant $\mathrm{D}$. To understand and explain why cloud service providers will adopt CSC, we ground our research on the TOE framework and the DOI theory.

\subsection{Technology-Organization-Environment Framework}

The TOE framework [29] describes which aspects in an organization's context influence the implementation and adoption of innovations. The three contexts that influence the technological innovation decision making are the organizational, environmental and technological context. First, the organizational context includes characteristics and resources of an organization that have an impact on innovation adoption. These include formal and informal linking structures between employees, internal communication processes, the size of the organization, and slack resources. Second, the environmental context comprises factors like the regulatory environment, the structure of the industry and the presence or absence of technology service providers. The structure of the industry could influence the innovation for example in case of intense competition that urges organizations to adopt an innovation. Finally, the technological context of the TOE framework includes all technological aspects that are relevant to the organization [29]. In this study, the technological context relates to the unique characteristics of the CSC innovation, and we therefore ground our study on the DOI theory that deals with characteristics of innovative technologies.

\subsection{Diffusion of Innovations Theory}

DOI theory [22] tries to explain how, why, and at what rate new ideas and technologies spread. A central concept of the DOI theory is the diffusion process, in which an innovation is communicated through certain channels, over time, among the members of a social system. Consequently, four key elements of DOI theory determine the characteristics of the diffusion process of an innovation: innovation, time, social system, and communication channels. An innovation is any idea, object, or practice that is perceived as new by the members of a 
social system. DOI theory argues that potential users make decisions to adopt or reject an innovation based on beliefs they form about the innovation. Time relates to the relative speed with which an innovation is adopted by members of the social system. The social system consists of individuals or organizations that share a common culture and are potential adopters of the innovation. Communication channels are the means by which information is transmitted to or within the social system. DOI theory proposes five major characteristics of an innovation that influence organization's adoption intention: relative advantage, compatibility, complexity, observability and trialability [22]. Relative advantage refers to the degree to which an innovation is perceived as being better than its precursors. Compatibility is the degree to which an innovation is perceived as consistent with existing sociocultural values and beliefs, previously introduced ideas, and needs of potential adopters. Complexity is the degree to which an innovation is perceived as relatively difficult to understand and use. Observability is the degree to which the results of an innovation are visible to others. Trialability is the degree to which an innovation can be tested before adoption.

\section{Research Model}

Figure 1 shows our research model to analyze organizational adoption intention of CSC in the context of cloud service providers. The model is divided into drivers and inhibitors of provider's adoption intention. We ground our model on the DOI theory and TOE framework to identify important drivers and inhibitors. In contrast to DOI theory, we excluded trialability because at the current stage of CSC there are no test versions available as CSC cannot be tested easily beforehand and it initially requires high efforts and expenditures.

\subsection{Adoption Drivers}

Relative advantage refers to the degree to which an innovation is perceived as being better than its precursors (i.e., resolving existing problems, or savings in time and effort) [22]. Providers must be motivated and have the expertise to participate in CSC. To motivate them, perceived advantages must be higher than perceived inhibitors. Cloud providers can achieve various advantages by CSC adoption, for example they receive ongoing feedback about offered services and are able to improve their service and risk management. In addition, cloud service providers can enhance transparency about their service for their customers, thus they might achieve competitive advantages. Hence, we hypothesize:

$H 1_{a}$ : Perceived relative advantages enhance provider's adoption intention of CSC.

Observability is the degree to which the results of an innovation are visible to others $[16,22]$. The results of some innovations can be easily observed and communicated to members of a social system, whereas some innovations are difficult to describe to others. In the context of CSC, cloud service customers are directly able to see that a service provider is continuously certified which leads to a high observability. A high observability might enhance the awareness about CSC which leads to higher adoption intentions. Thus:

$H 1_{b}$ : Perceived observability enhances provider's adoption intention of CSC.

Finally, the TOE framework highlights that an organization should consider their environmental context. We therefore focus on environmental factors, comprising competitive and customer pressures that might foster providers' adoption intention.

Competitive pressure is defined as pressure that is perceived from several sources within the competitive environment surrounding the organization, which forces an organization to adopt an innovation to stay competitive [4]. Adoption of CSC might increase competitiveness of providers because they might achieve competitive advantages as CSC increases cloud service transparency for customers. Thus, using CSC might result in a differentiation towards other competitors. Consequently, if the competitive pressure is high, it could be a driver for CSC adoption intention, and we postulate:

$H 1_{c}$ : Competitive pressure enhances provider's adoption intention of CSC.

Customer pressure is defined as the pressure that is perceived from customers, which forces an organization to adopt an innovation to fulfill customers' needs [17]. Customers report that cloud services are like black boxes as customers lack control of the services and transparency about service operation, leading to high customer uncertainty [31]. This uncertainty leads customers to demand innovative means that increase transparency and prove that cloud services are continuously reliable to make sure their business process for daily business are guaranteed. CSC could reduce these uncertainties and satisfy customers' needs by checking cloud services continuously. Hence, we hypothesize:

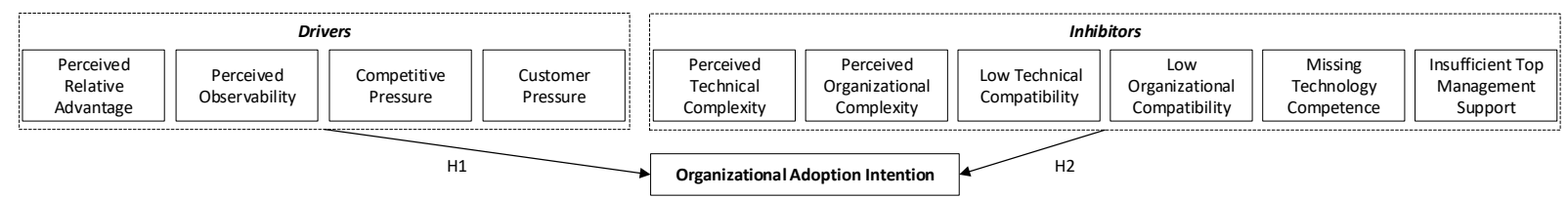

Figure 1. Research model 
H1 $1_{d}$ Customer pressure enhances provider's adoption intention of CSC.

\subsection{Adoption Inhibitors}

The introduction of a new innovation typically requires the organization to integrate (expensive) hardand software into its existing IT infrastructure, and can be intimidating for organizational employees, particularly if it requires them to change their existing business practices or to acquire new skills [22]. Thus, organizations face both technical as well as organizational complexity when adopting an innovation. A consistent finding from the technology diffusion literature is that technological complexity is a significant factor inhibiting implementation and adoption success [30]. In the context of CSC, providers are required to set up a comprehensive monitoring and auditing infrastructure because they must ensure that all certification-relevant data is available, up-to-date and accurate. Likewise, they need to provide secure data exchange interfaces to provide certification authorities with relevant data. The resulting effort and costs might hamper adoption of CSC:

$\mathrm{H} 2_{a}$ : Perceived technical complexity hampers provider's adoption intention of CSC.

Organizational complexity is the degree to which an innovation is perceived as relatively difficult to understand and use regarding potential users of the innovation $[5,22]$. Organizational aspects cover for example appreciation of employees by using CSC. If employees have no difficulties in understanding how to participate in CSC and participation is easy, the organizational complexity of CSC can be regarded as low. In contrast, higher difficulties in learning to participate CSC might hamper the adoption intention. Hence:

$\mathrm{H} 2_{b}$ : Perceived organizational complexity hampers provider's adoption intention CSC.

Further on, compatibility enhances diffusion of an innovation since an innovation that is more compatible is less uncertain to the potential adopter [3, 22]. Similar to complexity, compatibility can be further differentiated into technical and organizational compatibility. Technical compatibility focuses on whether the innovation fits to existing soft- and hardware. In case there is a need for cloud service providers to set up new (expensive) hard- or software to fulfill CSC requirements and to participate in CSC processes, a low technical compatibility is present. This situation might hamper the adoption of CSC. Hence, we propose:

$\mathrm{H} 2_{c}$ : A low technical compatibility hampers provider's adoption intention of CSC.

Organizational compatibility is the degree to which an innovation is perceived as consistent with the existing organizational values and beliefs, previously introduced ideas, and needs of potential adopters [22]. In this study, the organizational compatibility describes the ease to participate in CSC in particular. If employees of cloud service providers have to change their existing way of working, it will result in a low organizational compatibility. We argue that a low compatibility of the innovation with an organization leads to a lower adoption intention. Hence, we hypothesize:

$\mathrm{H} 2_{d}$ : A low organizational compatibility hampers provider's adoption intention of CSC.

Following the TOE framework, organization's capabilities might be limited in regard to meet the requirements of an innovation, and thus might lead to additional inhibitors. We therefore focus on organizational factors including organization's technology competence and top management support.

Technology competence is the degree technical knowledge available in the organization such as IT skills and IT infrastructure. These skills not only include current technical knowledge but also the ability to deploy, use, and manage the available IT infrastructure [10]. IT skills are mainly held by the employees that work in the IT department of an organization. In the case of CSC adoption, the need for technology competence might be high as cloud service providers need to set up comprehensive a monitoring and auditing infrastructure that exhibits a high degree of complexity. A compensation of a low technology competence might result in additional expenses, for example, in recruiting new employees. Hence, we propose:

$\mathrm{H} 2_{e}$ : A low technology competence hampers provider's adoption intention of CSC.

Top management support is the degree to which the top management of an organization provides the vision, initiative, support and commitment around the innovation [11]. The top management consists of people that work in an organization as the $\mathrm{CEO}, \mathrm{CFO}, \mathrm{CIO}$ and other senior-level business executives. In the case of CSC, service provider's decision to participate in a CSC involves a strategic and organizational wide decision. Consequently, an insufficient top management support has a negative effect on the adoption intention. Based on this assumption we propose:

$\mathrm{H} 2_{f}$ : Insufficient top management support hampers provider's adoption intention of CSC.

\section{Research Method}

\subsection{Survey Design}

The hypothesized research model is tested empirically by using an online survey. The survey consisted of six steps. In a first step, we asked subjects questions regarding their perception about current certification reliability among others to control for confounding effects 
that might have an impact on provider's adoption intention. Second, we introduced the concept of CSC by providing a detailed definition of CSC, summarizing reasons why traditional certifications are not sufficient in the context of cloud services, and finally briefly outlined how CSC works in comparison to traditional approaches. We tried to provide only general information about CSC to enable subjects to freely think about what actually is required to participate in CSC. Third, we requested subjects to imagine that their company is deciding whether they should participate in CSC. In this decision process, subjects were told to be a part of the project team that assesses several CSC offerings of certification authorities. We introduced a fictive CSC offering called 'InternationalCloudCert'. This offering comprised details about exemplary certification requirements that were checked during the CSC, including availability and vulnerability checks. In addition, we visualized CSC results by providing a draft of a dashboard depicting information about the certification status. After introducing the scenario, subjects had to decide whether their company should adopt CSC to measure our dependent variable. We then measured each independent variable. Finally, we asked for demographic information about the subjects and their company.

\subsection{Measurement}

The survey instrument used validated scales from the literature for our constructs. The survey items were measured using seven-point Likert scales. Survey instrument is summarized in the appendix. We adopted provider's adoption intention from [23] and [26]. The items for measuring relative advantage and perceived organizational complexity were adapted from [5]. We measured perceived technical compatibility of CSC and top management support by adopting an operationalization from [3]. In addition, we measured the perceived organizational compatibility by adopting measures from [8]. The items for measuring perceived organizational complexity were adapted from [5]. To measure perceived technical complexity we rely on measures from [7]. We measured perceived observability of CSC based on measures from [16]. Measures from [10] were used to measure technology competence of cloud service providers. Competitive pressure and customer pressure are measured by using measures from [4]. Items have been rephrased to meet the CSC context. For example, one organizational adoption intention item measures "To what extent is your group committed to the use of the above tool?" [23] and was rephrased to "Your company is committed to participate in continuous certification". While we did not identify suitable items for customer pressure, we adapted the measurements from related research on competitive pressure and rephrased them accordingly. For instance, the item "Please rate the pressure to adopt EDI placed on your organization by your competitors " [4] was rephrased to measure customer pressure: "Pressure to meet continuously technical, security, and privacy requirements is demanded by your main customers."

We controlled whether provider's size (number of employees) has an impact on adoption intention. To control for the current interest, faith and trust in thirdparty certifications we operationalized certification interest by adapting measurements from [20]. We used environmental turbulence to measure whether subjects would characterize cloud services environments as highly dynamic. The measurements were adapted from [19]. Finally, we controlled whether provider's market orientation (Business to Business (B2B) vs. Business to Consumer (B2C)), cloud service model (software, platform or infrastructure as a service) or cloud service deployment model (public, private, hybrid or community) have an impact on provider's adoption intention.

\subsection{Data Analysis}

To conduct the survey, we defined our target group as follows. We searched for experts (e.g., managers, technicians) that work for a cloud service provider. We included organizations that offer all kind of cloud services, including platform, software and infrastructure as a service, and public, private, community and hybrid cloud services as participating in CSC is independent of the cloud service deployment or service model. To acquire survey subjects, we heavily searched for provider websites. Based on this extensive online search we found 4,160 unique email addresses from cloud service providers. In addition, we worked together with a major certification authority that is offering cloud service certifications across Europe to distribute our survey. Finally, we rely on social media platforms (e.g., LinkedIn, XING etc.), visited an internationally represented computer expo for getting in touch with providers, and rely on personal contacts from previous research projects. We used snowballing sampling techniques, and thus requested survey participations to forward the survey to potential employees. Considering the feedback we received from subjects, this technique has proven to be very successful as many organizations forwarded our survey. Subjects were reminded to participate in our survey after one week which heavily increased response rate. We achieved 826 unique survey views, 191 survey participations and finally 115 complete datasets.

The subjects who participated in our survey were mostly male (84\%), 45 years old on average (minimum 24 years, maximum 63 years), and work in the position of a company founder, $\mathrm{CEO}, \mathrm{CFO}, \mathrm{CIO}$ or comparable (37\%), top management (16\%), head of department 
Table 2. Construct reliability and correlations

\begin{tabular}{|c|c|c|c|c|c|c|c|c|c|c|c|c|c|c|c|}
\hline Construct & $\overline{C R}$ & AVE & \multicolumn{13}{|c|}{ Inter-construct Correlations } \\
\hline & & & $(1)$ & $(2)$ & (3) & $(4)$ & (5) & $(6)$ & $(7)$ & $(8)$ & (9) & (10) & $(11)$ & $(12)$ & $\overline{(13)}$ \\
\hline (1) Adoption Intention & .93 & .82 & .904 & & & & & & & & & & & & \\
\hline (2) Certification Interest & .89 & .72 & .403 & .849 & & & & & & & & & & & \\
\hline (3) Competitive Pressure & .95 & .83 & .651 & .396 & .909 & & & & & & & & & & \\
\hline (4) Customer Pressure & 1. & 1. & .198 & .124 & .375 & 1. & & & & & & & & & \\
\hline (5) Enviro. Turbulence & .87 & .57 & .178 & .322 & .402 & .099 & .753 & & & & & & & & \\
\hline (6) Mgmt. Support & .90 & .75 & -.705 & -.388 & -.600 & -.254 & -.221 & .863 & & & & & & & \\
\hline (7) Observability & .92 & .73 & .693 & .251 & .678 & .334 & .225 & -.604 & .856 & & & & & & \\
\hline (8) Org. Compatibility & .94 & .89 & -.595 & -.136 & -.511 & -.167 & -.099 & .582 & -.584 & .941 & & & & & \\
\hline (9) Org. Complexity & .92 & .75 & -.648 & -.171 & -.566 & -.238 & -.174 & .570 & -.720 & .634 & .867 & & & & \\
\hline (10) Relative Advantage & .93 & .76 & .759 & .255 & .720 & .186 & .245 & -.610 & .633 & -.566 & -.598 & .873 & & & \\
\hline (11) Tech. Compatibility & .95 & .91 & -.486 & -.154 & -.358 & -.188 & -.038 & .555 & -.474 & .581 & .547 & -.400 & .954 & & \\
\hline (12) Tech. Competence & .85 & .66 & -.129 & -.177 & -.219 & -.137 & -.405 & .240 & -.117 & .035 & .194 & -.063 & .058 & .810 & \\
\hline (13) Tech. Complexity & .94 & .83 & .265 & .281 & .264 & .189 & .119 & -.256 & .220 & -.102 & -.091 & .221 & -.120 & -.109 & .912 \\
\hline
\end{tabular}

$(12 \%)$, team leader $(17 \%)$, staffer $(8 \%)$ or other $(2 \%)$. The company they work for is based in Germany (33\%), Sweden (4\%), Denmark (3\%), Switzerland (3\%). Their service offerings were characterized by Business-toBusiness market $(80 \%)$, Business-to-Customer market $(15 \%)$, Software $(67 \%)$, Platform $(35 \%)$ or Infrastructure as a Service (43\%), Public (43\%), Private (44\%), Community (10\%) and Hybrid Cloud (37\%).

To test our hypothesis, we used partial least squares structural equation modeling (PLS-SEM) using SmartPLS 3.2.6 [21]. First, we assessed our model in regard to internal consistency, convergent validity and discriminant validity. Table 2 presents the construct measurements, including composite reliability (CR), average variance extracted (AVE), and correlations between latent variables. Calculations of CR demonstrate good internal consistency because each construct shows a value greater than .7, which exceeds the recommended threshold [18]. The AVE for each construct is greater than the suggested minimum of .5 [6]. We assessed the convergent validity by examining individual indicator loadings between the indicator and its corresponding underlying factor. Reliable indicators should have a loading of .7 or higher; in cases when convergence criteria are fulfilled (AVE should exceed .5), loadings below .7 but equal to .6 or higher are acceptable as well. We dropped three items of environmental turbulence (see Table 3 in the appendix). Afterwards, all indicators fulfill the minimum loading requirements. Further, we identified two potential discriminant validity problems between customer and competitive pressure as well as between relative advantage and adoption intention as the more rigorous criterion for assessing discriminant validity, the heterotrait-monotrait (HTMT) ratio of correlations $[9,33]$, shows a HTMT value higher than the recommended threshold of 0.85 . We therefore dropped one item in customer pressure and adoption intention based on an analysis of item cross loadings (see Table 3 in the appendix). Afterwards, the model shows good discriminant validity because the square root of each construct's AVE (highlighted in Table 2 on the diagonal) exceeds the inter-construct correlations, and all HTMT values are below the recommended threshold.

We tested our structural model by evaluating the direct effects as well as the explained variances $\left(\mathrm{R}^{2}\right)$ (see Figure 2). The significance of the structural path estimates was assessed using the bootstrapping algorithm with 5,000 sub-samples and bias-corrected and accelerated confidence intervals [21].

Data analysis reveals that provider's adoption intention is strongly driven by perceived relative advantages $(.458 ; \mathrm{p}<.01 ;[.245 ; .672])$ and observability (.205; $\mathrm{p}=.037$; $[.017 ; .404])$, supporting H1a and H1b. Contrary to our assumptions, customer pressure (-.039; $\mathrm{p}=.56 ;[-.167 ; .099])$ and competitive pressure $(-.017$; $\mathrm{p}=.88 ;[-.227 ; .206])$ do not have any significant impact on adoption intention, rejecting $\mathrm{H} 1 \mathrm{c}, \mathrm{d}$.

In regard to the influence of inhibitors on provider's adoption intention, we only identify a weak negative effect of insufficient top management support on adoption intention $(-.184 ; \mathrm{p}=.099 ;[-.392 ; .034])$, weakly supporting $\mathrm{H} 2 \mathrm{f}$. We find no evidence in our data that technical $(.044 ; \mathrm{p}=.50 ;[-.086 ; .166])$ or organizational

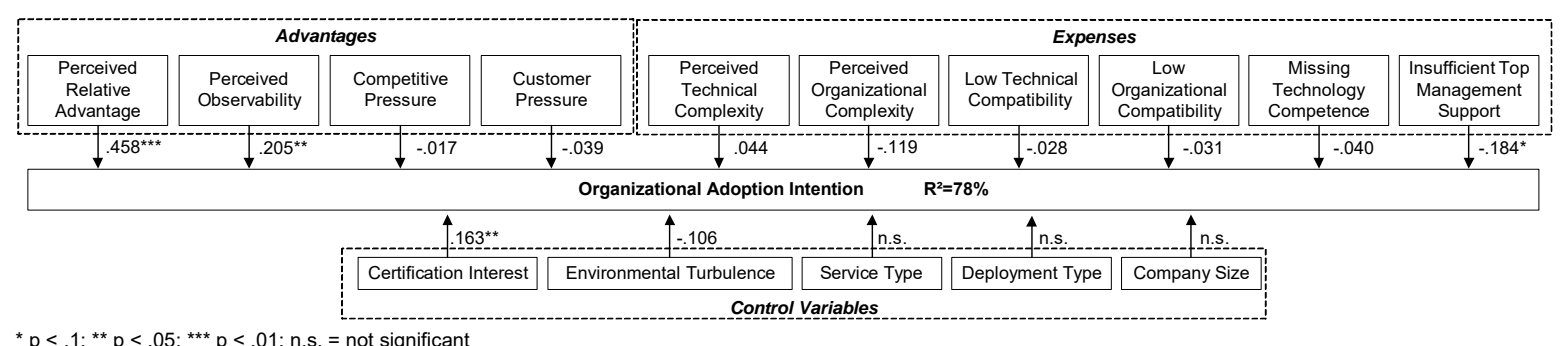

Figure 2. Path model 
complexity $(-.119 ; \mathrm{p}=.20 ;[-.310 ; .062])$, a low technical $(-.028 ; \mathrm{p}=.74 ;[-.183 ; .151])$ or organizational compatibility $(-.031 ; \mathrm{p}=.76 ;[-.245 ; .147])$, or a missing technology competence $(-.040 ; p=.62 ;[-.186 ; .131])$ have an impact on provider's adoption intention, rejecting $\mathrm{H} 2 \mathrm{a}, \mathrm{b}, \mathrm{c}, \mathrm{d}$,e. Concerning our control variables, we did not identify any significant effect of the service (Software, Platform or Infrastructure as a Service) or deployment model (public, private, hybrid, community) of the offered cloud service, or provider's market orientation (B2B, B2C). Similarly, provider's size or perceived environmental turbulence of the cloud computing environment has no significant effect. Nevertheless, provider's general interest in certifications has a significant positive influence on the adoption intention (.163; $\mathrm{p}=.021 ;[.032 ; .316])$.

\section{Discussion}

Our findings confirm that cloud service providers are willing to adopt CSC (adoption intention mean $=4.42$ ). Considering that CSC is a new innovation that has been under research since the last years, and thus widespread knowledge about CSC is still lacking, this finding highlights the importance and potential of CSC to change the cloud service environment.

Data analysis revealed that our theoretical model is able to explain $78 \%$ of the variance $\left(\mathrm{R}^{2}\right)$ of cloud service provider's adoption intention, and thus provides a good explanation. In particular, our results show that perceived advantages strongly drive the adoption intention of cloud service providers. And hence, cloud service providers agree that participating in CSC will help them to achieve many benefits. This finding underlines the importance of the innovative development of CSC, and the need to enhance its diffusion. In addition, observability of participation is also a very important driver of CSC. A high observability shows that CSC is visible to others and employees of cloud providers could easily explain why CSC is beneficial or not. The missing significance in customer and competitive pressure indicate that there is currently no high impact of customers and competitors regarding CSC adoption. This finding is not surprising as CSC is on its early diffusion stage, and competitors might not be aware of CSC, and customers have just started to demand for continuously secure cloud services. In line with DOI theory, the influence of these factors might get stronger in later adoption phases as CSC is spreading through the environment [22].

In regard to the impact of inhibitors, our survey revealed a surprising finding as none of the factors are significant. A reason for this could be that CSC was so far tested in prototypes and research projects only. Provid- ers might lack profound knowledge about CSC complexity, and which expenses might emerge by adopting CSC. A precise description of potential expenses (e.g., cost of monitoring and auditing software) due to a high complexity of CSC might decrease the lack of information, and thus might increase influence of these inhibitors on adoption intention. In addition, providers reported that they possess a high technical competence (mean of technical competence 5.9 (not reversed)), and that their existing technical infrastructure will be compatible with CSC processes (mean of technical compatibility 4.4 (not reversed)). Consequently, they might feel confident when facing the requirements of participating in CSC, and might not fear strong inhibitors.

Our study results in several implications for research. We derived and tested a theoretical model explaining the CSC adoption intention of service providers, and thereby deepened the knowledge about what drives providers' adoption intention and addressed the prevalent research gap. This is particularly valuable as previous literature has mostly taken an (internal) auditor perspective when analyzing stakeholders' adoption intention. In line with research on DOI theory, we find evidence that relative advantages have a strong influence on the adoption intention [22]. Contrary to our assumptions, inhibitors are not relevant in our context, and future research is required to deepen the understanding about this counterintuitive finding.

For practice, our study highlights that certification authorities should promote expected advantages of CSC to motivate cloud service providers to participate. In addition, providers and certification authorities have to ensure that results of participating in CSC are visible for cloud service customers and the environment. While we did not identify an impact of competitive pressure, cloud service providers have to evaluate whether they should be early adopters of CSC to achieve first mover advantages and improve their market position.

Nevertheless, our study has some limitations. First, we surveyed various cloud service providers from different countries (mostly from Europe). On the one hand, this helped us to get a holistic view of different cloud service providers across the globe. On the other hand, findings might not be generalizable to other countries like the US as they might have a different view of CSC due to their local market perceptions or regulatory landscape. Moreover, we briefly described the concept of CSC during our survey to enable subjects to freely think about what actually is required to participate in CSC. This approach, however, might result in an interpretation bias as subjects might differ in their imagination about CSC.

Future work might focus on whether certification authorities are willing to provide required CSC services as 
these service offerings form a prerequisite for a diffusion of CSC. In addition, future work might evaluate whether providers are actually able to meet CSC complexity as they possess a high technical competence and as available systems seem to be compatible with the requirements of participating in CSC. Finally, follow-up studies should also analyze customers' and auditors' perspective to deepen understanding of CSC adoption.

\section{Conclusion}

In this study, we analyzed factors that influence the adoption of CSC in the context of cloud service providers. In particular, we focused on the trade-off between achieving advantages and participating in a complex and effortful CSC process. Overall, our findings highlight that the relative advantage and observability strongly influence the adoption intention of cloud service providers and outweigh potential inhibitors.

\section{Appendix}

Table 3. Construct Operationalization Organizational Adoption Intention $[23,26]$
${ }^{*}$ Your company intends to participate in a continuous certification. It is likely that your company would take some steps to participate in a continuous certification in the future.

Your company is convinced to participate in continuous certification. Your company is committed to participate in continuous certification. Perceived Relative Advantage [5]

Participating in a continuous certification improves your company's cloud service performance.

Participating in a continuous certification increases your company's productivity.

Participating in a continuous certification enhances your company's effectiveness on providing cloud services.

Participating in a continuous certification is useful for your company. Low Technical Compatibility [3]

(R) Participating in a continuous certification is compatible with existing software systems in your company.

${ }^{(R)}$ Participating in a continuous certification is compatible with existing hardware in your company.

Low Organizational Compatibility [8]

${ }^{(R)}$ Participating in a continuous certification fits well with the way employees work.

${ }^{(R)}$ Participating in a continuous certification is compatible with all aspects of an employee's work.

Perceived Organizational Complexity [5]

${ }^{(R)}$ Learning to participate in a continuous certification is easy for your company's employees.

(R) Your company's employees' participation in a continuous certification is clear and understandable.

${ }^{(R)}$ It is easy for your company's employees to become skillful at participating in a continuous certification.

${ }^{(R)}$ Your company's employees find it easy to participate in a continuous certification.

Perceived Technical Complexity [7]

The technical requirements for participating in a continuous certification (e.g. need to set up comprehensive monitoring and auditing infrastructure) make it complex.

A continuous certification depends on a sophisticated integration of technology component (e.g. need to set up comprehensive monitoring and auditing infrastructure).

There is considerable technical complexity when participating in a continuous certification.

Perceived Observability [16]
The employees of your company believe they can easily communicate the consequences of participating in a continuous certification to their customers.

The results of participating in a continuous certification are apparent to the customers of your company.

The employees of your company have no difficulty to explain why participating in a continuous certification may be beneficial.

Participating in a continuous certification is very visible to your custom-

ers.

Missing Technology Competence [10]

${ }^{(R)}$ A high amount of time and effort goes into developing and maintaining IT skills, compared to other aspects of your business.

${ }^{(R)}$ Your company has a high overall competence level in IT skills.

${ }^{(R)}$ IT skills are of high importance for your company.

Insufficient Top Management Support [3]

${ }^{(R)} A$ continuous certification will receive strong active support from your company's top management.

(R) Your company's upper management will provide adequate financial

and other resources to participate in a continuous certification.

${ }^{(R)}$ The success of participating in a continuous certification will be due to the active championing by your company's key senior management per-

son(s).

Competitive Pressure [4]

Your company will experience competitive pressure to participate in a continuous certification in the future.

In your industry, the participation in continuous certification will be helpful in allowing a company to remain competitive.

Customer Pressure (following [4])

${ }^{*}$ Customers (will) prefer cloud services from companies that participate in a continuous certification.

Pressure to meet continuously technical, security, and privacy require-

ments is demanded by your main customers.

Certification Interest [20]

Your company is interested in third-party IT certifications.

Your company generally has faith in third-party IT certifications.

Your company generally trusts third parties that award IT certifications.

Environmental Turbulence [19]

The environment in our service area is continuously changing.

* Environmental changes in our service area are very difficult to forecast.

The technology in this service area is changing rapidly.

Technological breakthroughs provide big opportunities in this service area.

In our kind of business, customers' preferences for services and func-

tionalities change a lot over time.

* Marketing practices in our service area are constantly changing to keep

pace with the market and competitors.

Launches of new services and functionalities are very frequent in this

market.

* There are many regulatory or governmental changes that affect our services.

* Item was dropped during model assessments;

(R) Item was reversed scaled.

\section{References}

[1] Abou-El-Sood, H., A. Kotb, and A. Allam, "Exploring Auditors' Perceptions of the Usage and Importance of Audit Information Technology", INT J Auditing, 19(3), 2015, pp. 252-266.

[2] Bierstaker, J., D. Janvrin, and Lowe, D. Jordan, "What Factors Influence Auditors' Use of Computer-assisted Audit Techniques?", ADV in ACC, 30(1), 2014, pp. 67-74.

[3] Bradford, M. and J. Florin, "Examining the Role of Innovation Diffusion Factors on the Implementation Success of Enterprise Resource Planning Systems", INT J of ACC INF SYST, 4(3), 2003, pp. 205-225.

[4] Chwelos, P., I. Benbasat, and A.S. Dexter, "Empirical Test of an EDI Adoption Model", INF SYST RES, 12(3), 2001, pp. 304-321. 
[5] Davis, F.D., "Perceived Usefulness, Perceived Ease of Use, and User Acceptance of Information Technology", MIS Q, 13(3), 1989, pp. 319-340.

[6] Fornell, C. and D.F. Larcker, "Evaluating Structural Equation Models with Unobservable Variables and Measurement Error", J of MARK RES, 18(1), 1981, pp. 39-50.

[7] Furneaux, B. and M. Wade, "An Exploration of Organizational Level Information Systems Discontinuance Intentions", MIS Q, 35(3), 2011, pp. 573-598.

[8] Hardgrave, B.C., F.D. Davis, and C.K. Riemenschneider, "Investigating Determinants of Software Developers' Intentions to Follow Methodologies", J MANAGE INF SYST, 20(1), 2003, pp. 123-151.

[9] Henseler, J., C.M. Ringle, and M. Sarstedt, "A New Criterion for Assessing Discriminant Validity in Variance-Based Structural Equation Modeling", J ACAD MARKET SCI, 43(1), 2014, pp. 115-135.

[10] Hulland, J., M.R. Wade, and K.D. Antia, "The Impact of Capabilities and Prior Investments on Online Channel Commitment and Performance", J MANAGE INF SYST, 23(4), 2007, pp. 109-142.

[11] Lee, S. and K.-j. Kim, "Factors Affecting the Implementation Success of Internet-based Information Systems", COMP HUM BEHAV, 23(4), 2007, pp. 1853-1880.

[12] Lins, S., P. Grochol, S. Schneider, and A. Sunyaev, "Dynamic Certification of Cloud Services: Trust, but Verify!", IEEE Security \& Privacy, 14(2), 2016, pp. 67-71.

[13] Lins, S., S. Schneider, and A. Sunyaev, "Trust is Good, Control is Better: Creating Secure Clouds by Continuous $\mathrm{Au}-$ diting", IEEE Transactions on Cloud Computing, forthcoming, 2016, pp. 1-14

[14] Lins, S., H. Teigeler, and A. Sunyaev, "Towards a Bright Future: Enhancing Diffusion of Continuous Cloud Service Auditing by Third Parties", in Proceedings of the ECIS 2016, Istanbul, Turkey, June 12-15. 2016.

[15] Lins, S., S. Thiebes, S. Schneider, and A. Sunyaev, "What is Really Going on at Your Cloud Service Provider?", in Proceedings of the HICSS 2015, Hawaii, USA, Januar 4-7. 2015: Kauai, Hawaii, USA.

[16] Moore, G.C. and I. Benbasat, "Development of an Instrument to Measure the Perceptions of Adopting an Information Technology Innovation", INF SYST RES, 2(3), 1991, pp. 192-222.

[17] Ngai, E.W.T., D.C.K. Chau, J.K.L. Poon, A.Y.M. Chan, B.C.M. Chan, and W.W.S. Wu, "Implementing an RFIDbased Manufacturing Process Management System", J ENG and TECHNOL MANAG, 29(1), 2012, pp. 112-130.

[18] Nunnally, J.C. and I.H. Bernstein, Psychometric Theory, 3rd edn., McGraw-Hill, New York, 1994.

[19] Pavlou, P.A. and O.A. El Sawy, "The "Third Hand"", INF SYST RES, 21(3), 2010, pp. 443-471.
[20] Ponte, E.B., E. Carvajal-Trujillo, and T. EscobarRodríguez, "Influence of Trust and Perceived Value on the Intention to Purchase Travel Online", Tourism MANAGE, 47, 2015, pp. 286-302.

[21] Ringle, C.M., S. Wende, and J.-M. Becker, "SmartPLS 3", SmartPLS GmbH, www.smartpls.com, Boenningstedt, Germany, 2015.

[22] Rogers, E.M., Diffusion of Innovations, 3rd edn., Free Press, New York, 1983.

[23] Sarker, S. and J.S. Valacich, "An Alternative to Methodological Individualism", MIS Q, 34(4), 2010, pp. 779-808.

[24] Schneider, S. and A. Sunyaev, "Determinant Factors of Cloud-sourcing Decisions", J INF TECHNOL, 31(1), 2016, pp. 1-31.

[25] Singh, K., P.J. Best, M. Bojilov, and C. Blunt, "Continuous Auditing and Continuous Monitoring in ERP Environments", J INFORM SYST, 28(1), 2013, pp. 287-310.

[26] Son, J.-Y. and I. Benbasat, "Organizational Buyers' Adoption and Use of B2B Electronic Marketplaces", J MANAGE INF SYST, 24(1), 2007, pp. 55-99.

[27] Stephanow, P. and C. Banse, "Evaluating the Performance of Continuous Test-based Cloud Service Certification", in 17th IEEE/ACM International Symposium on Cluster, Cloud and Grid Computing, 14-17 May. 2017.

[28] Sunyaev, A. and S. Schneider, "Cloud Services Certification", CACM, 56(2), 2013, pp. 33-36.

[29] Tornatzky, L.G., M. Fleischer, and A.K. Chakrabarti, The Processes of Technological Innovation, Lexington Books, Lexington, Mass., 1990.

[30] Tornatzky, L.G. and K.J. Klein, "Innovation Characteristics and Innovation Adoption-implementation", IEEE Trans on ENG MANAGE, EM-29(1), 1982, pp. 28-45.

[31] Trenz, M., J. Huntgeburth, and D. Veit, "The Flock in the Cloud - How Social Influence Processes Shape Cloud Service Relationships", in Proceedings of the ICIS 2015, Fort Worth, Texas, December 13-16. 2015.

[32] Vasarhelyi, M.A., M. Alles, S. Kuenkaikaew, and J. Littley, "The Acceptance and Adoption of Continuous Auditing by Internal Auditors", Methodologies in AIS Research, 13(3), 2012, pp. 267-281.

[33] Voorhees, C.M., M.K. Brady, R. Calantone, and E. Ramirez, "Discriminant Validity Testing in Marketing: An Analysis, Causes for Concern, and Proposed Remedies", J ACAD MARKET SCI, 44(1), 2015, pp. 119-134.

[34] Yeh, C.-H., T.-P. Chang, and W.-C. Shen, "Developing Continuous Audit and Integrating Information Technology in E-business", in Proceedings of the APSCC 2008, Yilan, Taiwan, December 9-12. 2008. 